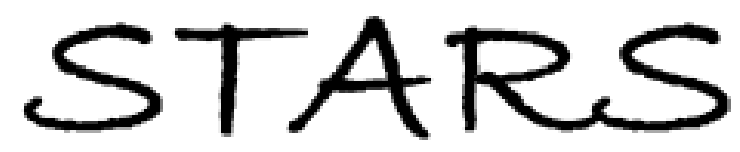

University of Central Florida

STARS

$1-1-1996$

\title{
Quasi-Phase Matched Surface Emitting Second Harmonic Generation In Poled Polymer Waveguides
}

\author{
A. Otomo \\ University of Central Florida \\ G. I. Stegeman \\ University of Central Florida \\ W. H. G. Horsthuis \\ G. R. Möhlmann
}

Find similar works at: https://stars.library.ucf.edu/facultybib1990

University of Central Florida Libraries http://library.ucf.edu

This Article is brought to you for free and open access by the Faculty Bibliography at STARS. It has been accepted for inclusion in Faculty Bibliography 1990s by an authorized administrator of STARS. For more information, please contactSTARS@ucf.edu.

\section{Recommended Citation}

Otomo, A.; Stegeman, G. I.; Horsthuis, W. H. G.; and Möhlmann, G. R., "Quasi-Phase Matched Surface Emitting Second Harmonic Generation In Poled Polymer Waveguides" (1996). Faculty Bibliography 1990s. 1707.

https://stars.library.ucf.edu/facultybib1990/1707

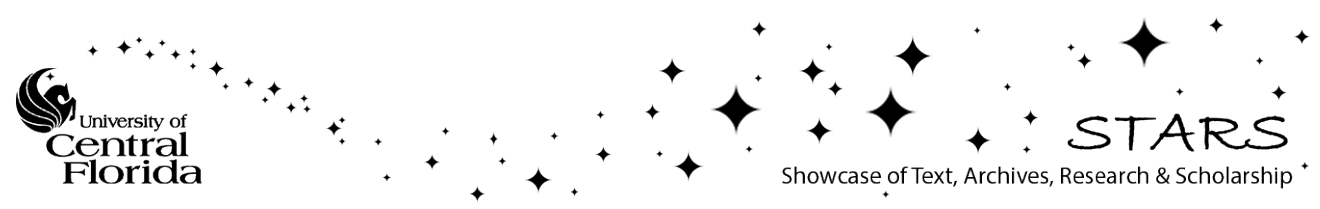




\section{Quasi-phase matched surface emitting second harmonic generation in poled polymer waveguides}

Cite as: Appl. Phys. Lett. 68, 3683 (1996); https://doi.org/10.1063/1.115742

Submitted: 06 February 1996 . Accepted: 22 April 1996 . Published Online: 05 August 1998

A. Otomo, G. I. Stegeman, W. H. G. Horsthuis, and G. R. Möhlmann

\section{ARTICLES YOU MAY BE INTERESTED IN}

Enhanced second-harmonic generation by counter-propagating guided optical waves Applied Physics Letters 38, 759 (1981); https://doi.org/10.1063/1.92152
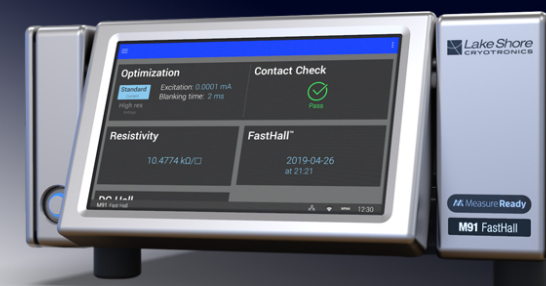

\section{Measure Ready} M91 FastHall ${ }^{\text {TM }}$ Controller

A revolutionary new instrument for complete Hall analysis See the video 0 


\title{
Quasi-phase matched surface emitting second harmonic generation in poled polymer waveguides
}

\author{
A. Otomo ${ }^{\text {a) }}$ and G. I. Stegeman \\ Center for Research and Education in Optics and Lasers, University of Central Florida, Orlando, \\ Florida 32816-2700 \\ W. H. G. Horsthuis and G. R. Möhlmann \\ Akzo Nobel Electronic Products, Arnhem, The Netherlands
}

(Received 6 February 1996; accepted for publication 22 April 1996)

\begin{abstract}
Quasi-phase matched (QPM) surface emitting second harmonic generation was demonstrated with nonlinear/linear multilayer waveguides in poled polymer based devices. The nonlinear/linear multilayer film was fabricated with a 4-dimethylamino-4'-nitrostilbene (DANS) side chain polymer and a cross-linkable clear polymer. Large efficiency improvement was observed with a combination of QPM and strong field parallel poling. (c) 1996 American Institute of Physics. [S0003-6951(96)01426-X]
\end{abstract}

Surface emitting second harmonic generation (SE-SHG) by the mixing of counterpropagating guided waves in poled polymers has been demonstrated in 4-dimethylamino-4'-nitrostilbene (DANS) side-chain polymer waveguides. ${ }^{1}$ Although the poling fields available at that time were small $(50 \mathrm{~V} / \mu \mathrm{m})$, large second harmonic conversion was observed compared to inorganic and semiconductor single layer devices. ${ }^{2,3}$ This is due to the large polymer second order nonlinearities and small refractive indices at the second harmonic wavelength. However, in the last few years, several experiments in GaAs based waveguides were reported and the conversion efficiency was improved dramatically by using a form of quasi-phase matching (QPM) in the transverse direction. ${ }^{3}$ A large efficiency improvement is also expected in organic polymers by employing both transverse QPM and the large poling fields now available. ${ }^{4}$

Phase matching is one of the most essential requirements for efficient harmonic generation of copropagating fundamental and harmonic beams. In the case of mixing of two counterdirected guided beams, the phase matching condition is always satisfied along the propagation direction, since the harmonic field is radiated out from the film surface. However, large phase mismatches occur along the transverse direction, i.e., perpendicular to the film surface. Transverse phase matching is often representing by maximizing the overlap integral $S$ which is expressed for the counterpropagating case as

$$
S=\int_{-\infty}^{\infty} \frac{d_{22}\left(x^{\prime}\right) f_{y}\left(x^{\prime}\right)^{2}}{\tilde{n}_{f}^{(2 \omega)}\left(x^{\prime}\right)} \exp \left(i k_{0}^{(2 \omega)} \widetilde{n}_{f}^{2 \omega}\left(x^{\prime}\right) x^{\prime}\right) d x^{\prime},
$$

where $f_{y}$ is the guided field distribution of the counterpropagating fundamental beams, $\tilde{n}_{j}^{2 \omega}$ is the complex refractive index of the film, and $d_{22}$ is the second order nonlinearity. The exponential term represents the spatial distribution of the phase of the generated second harmonic field. Since the generated second harmonic intensity is quadratic in $S$, the expected second harmonic signal can be estimated by investigating the overlap integral. The integrand oscillates across the waveguide varying from positive to negative values with

a)Electronic mail: akira@soliton.creol.ucf.edu a period corresponding to the second harmonic wavelength as shown in Fig. 1(a). Different $d_{22}$ distributions are shown below in that figure. In a single layer waveguide for which $d_{22}$ is constant, the resultant integral is small due to the de-
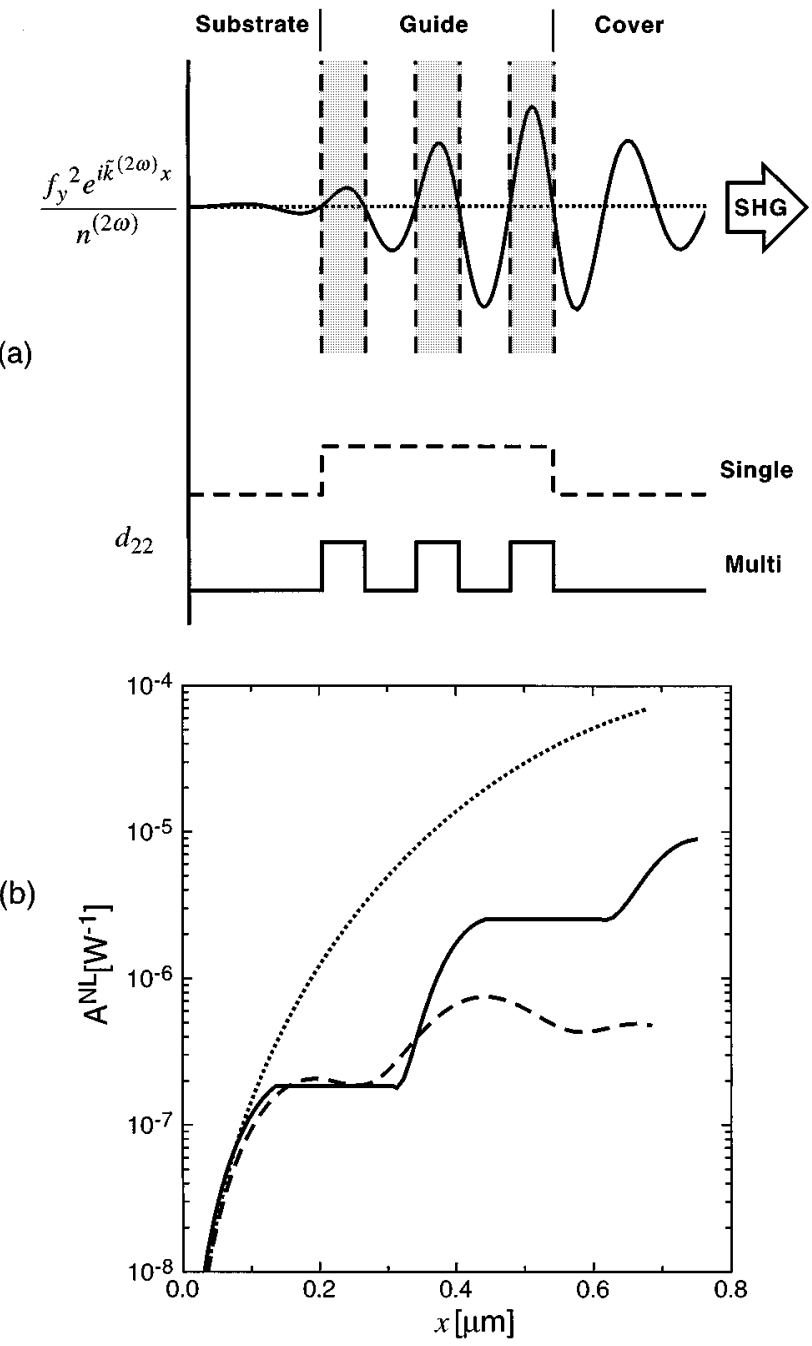

FIG. 1. Transverse quasiphase matching in surface emitting SHG; (a) schematic representation of the integrand in the overlap integral; (b) second harmonic growth through the film. (-) NL/L multilayer film, (---) single layer film; $(\cdots)$ perfectly phase-matched film. 


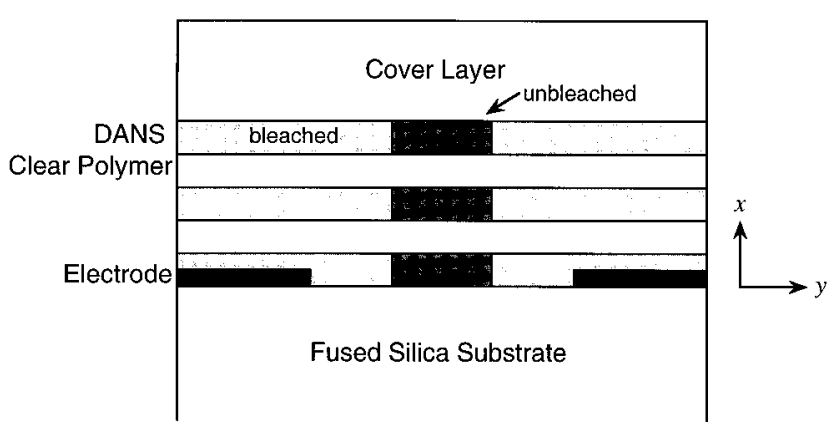

FIG. 2. Schematic of the cross section of the NL/L multilayer waveguide.

structive interference between the second harmonic waves generated from the different depths corresponding to successive half-wavelength regions [Fig. 1(b)]. The destructive interference can be partially compensated by modulating $d_{22}$ along the film depth, a form of quasi-phase matching. In previous semiconductor QPM experiments, the Al concentration (and therefore $d_{22}$ ) was modulated in AlGaAs waveguides. ${ }^{3,5}$ For poled polymer devices, three possible modulation methods have been proposed, specifically (a) a nonlinear/linear $(\mathrm{NL} / \mathrm{L})$ multilayer in which $d_{22}$ varies between zero and $+d$ [Figs. 1(a) and 1(b)] a reverse poling multilayer in which full modulation between $\pm d$ is constructed by using two different $T_{g}$ polymers, and (c) a heterophase multilayer where full modulation is achieved by utilizing the phase of the complex nonlinear coefficient. ${ }^{6} \mathrm{We}$ fabricated NL/L multilayer films successfully and QPM enhanced SHG was observed in poled polymer based SE-SHG devices. In a quasi-phase matched structure, the SH light grows monotonically towards the radiating film surface and an order of magnitude efficiency enhancement is expected compared to the single layer device, as shown in Fig. 1(b). Here $A^{\mathrm{NL}}$ is the nonlinear cross-section coefficient defined by $P^{(2 \omega)}=A^{\mathrm{NL}}(L / W) P_{+}^{(\omega)} P_{-}^{(\omega)}$ where $P^{(2 \omega)}$ is the radiated SH power, $P_{ \pm}^{(\omega)}$ are the counterdirected fundamental power, and $L$ and $W$ are the device interaction length and the channel waveguide width, respectively. Although complete phase matching is not achievable along the transverse direction, the maximum possible efficiency is one order of magnitude larger than for the NL/L QPM device.

Multilayer polymer films were fabricated by choosing the approximate combination of polymers and solvents which would not dissolve the successive cured polymers beneath. First, a film on DANS side-chain polymer was spincoated onto a fused silica substrate on which waveguide coupling gratings were first ion milled and poling aluminum electrodes deposited. A clear cross-linkable polymer with no polable chromophores was spun on top of the DANS layer and thermally cured to form cross linking. The cross-linked clear film was resistive to the solvent which was used to dissolve the DANS polymer. Chlorobenzene was used to dilute the clear polymer, since the DANS polymer is resistive to it. After spin coating successive layers consisting of three layers of the DANS film and two layers of the clear film, a $15 \mu \mathrm{m}$ thick cover layer also made of the clear polymer was fabricated to prevent arcing through the air in high field parallel poling as shown in Fig. 2. ${ }^{4}$ The thickness of each thin (a)

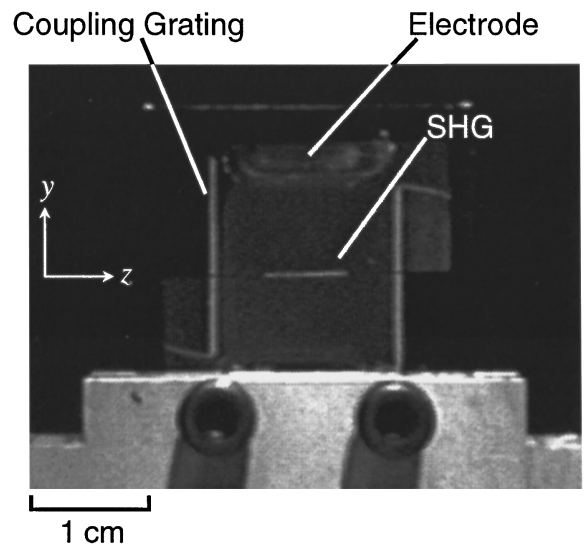

(b)
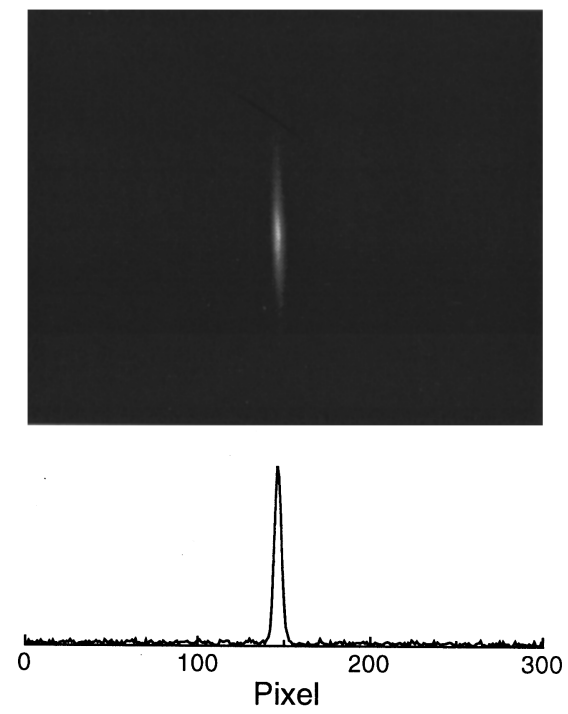

FIG. 3. Surface emitting SHG images taken by a CCD camera (a) at the image plane and (b) at the Fourier plane.

film corresponds to one-half of the second harmonic wavelength. Plane parallel poling was performed at just below the glass transition temperature of the DANS film $\left(T_{g}=142{ }^{\circ} \mathrm{C}\right)$ where the DANS molecules are rotationally mobile. Multilayer NLO waveguides were successfully poled with applied fields of over $300 \mathrm{~V} / \mu \mathrm{m}$. The poled films possess a nonlinearity of $d_{22}=150 \mathrm{pm} / \mathrm{V}$ at $1.06 \mu \mathrm{m}$. A channel waveguide structure including tapered mode couplers was defined by photobleaching through a mask using a $\mathrm{Hg}$-arc lamp before making the thick cover layer. ${ }^{7}$

Figure 3 shows surface emitting SHG from a five layer NL/L multilayer device imaged by a CCD camera under bright lighting conditions. The two grating couplers are separated by $15 \mathrm{~mm}$, one at each end of the substrate and the poling electrodes are separated by a $20 \mu \mathrm{m}$ gap inside of which the channel waveguide was fabricated. The tapered mode couplers, located at the coupling edge of the input gratings, are designed to compress the waveguide width from the $30 \mu \mathrm{m}$ coupling region to the $4 \mu \mathrm{m}$ channel over a $2 \mathrm{~mm}$ length. The input fundamental pulses were beam split halves of a 100 ps pulse from a $Q$-switched $(500 \mathrm{~Hz})$ mode locked $(76 \mathrm{MHz}) \mathrm{Nd}^{3+}$ : YAG laser. The pulse width is larger than the device length and the pulses are adjusted to overlap in the middle of the waveguide. Since the SHG radiation diffracts with an angle of only $70 \mu \mathrm{rad}(\propto 1 / L)$ along the $z$ axis, only 
part of the streak which corresponds to the size of the aperture of the camera, can be seen [Fig. 3(a)]. An image at the Fourier plane in Fig. 3(a) shows a single narrow line which verifies single mode operation for the waveguide at the fundamental wavelength. Another experiment with two fundamental modes clearly showed three spots corresponding to the mixing between the $\pm \mathrm{TE}_{00}$ and $\pm \mathrm{TE}_{01}$ modes. The measured nonlinear cross section ${ }^{8}$ of the multilayer device is $A^{\mathrm{NL}}=2.3 \times 10^{-6} \mathrm{~W}^{-1}$ and the conversion efficiency is $\eta=P^{(2 \omega)} / L P_{+}^{(\omega)} P_{-}^{(\omega)}=0.6 \% / \mathrm{W} \mathrm{cm}$. With the larger nonlinearities available near-resonance and a narrower waveguide such as $W=1 \mu \mathrm{m}$, a power conversion efficiency $\eta=100 \%$ / $\mathrm{W} \mathrm{cm}$ can be obtained. The measured cross section is $20 \%$ of the theoretically calculated value. Since the waveguide propagation losses used to evaluate the cross section were characterized for the single layer device, there can be increased losses due to the multilayer structure. For example, strong stray scattering was observed in the taper region, especially at the end of the taper. Another possible reason for the discrepancy is inhomogeneity of the nonlinearity across the poling electrode gap. Although the inhomogeneity and the influence of the charge injection were found to be small for the film poled at $50 \mathrm{~V} / \mu \mathrm{m},{ }^{4}$ a larger influence of charge injection can occur for films poled at higher fields. Since the measured nonlinearity for the parallel poled film is the averaged value across the gap, the nonlinearity in the middle of the gap where the channel waveguide is located can be over- estimated. We have not investigated the lifetime of device activity in detail, however, SH efficiency measured for films 11 months after poling decreased less than $15 \%$ from the original value.

In summary, quasi-phase matched surface emitting SHG was demonstrated in poled polymer based devices using NL/L multilayer films. A large improvement in the efficiency was observed and even higher conversion efficiency is expected with optimum designed devices.

The authors acknowledge the support of the Air Force Office of Scientific Research (F49620-95-1-0147). We also thank J. Ross for preparing the coupling gratings and the poling electrodes.

${ }^{1}$ A. Otomo, S. Mittler-Neher, C. Bosshard, G. I. Stegeman, W. H. G. Horsthuis, and G. R. Möhlmann, Appl. Phys. Lett. 63, 3405 (1993).

${ }^{2}$ R. Normandin and G. I. Stegeman, Appl. Phys. Lett. 40, 759 (1982).

${ }^{3}$ R. Normandin, R. L. Williams, and F. Chatenoud, Electron. Lett. 26, 2088 (1990); R. Normandin, S. Létourneau, F. Chatenoud, and R. L. Williams, IEEE J. Quantum Electron. QE-27, 1520 (1991).

${ }^{4}$ A. Otomo, G. I. Stegeman, W. H. G. Horsthuis, and G. R. Möhlmann, Appl. Phys. Lett. 65, 2389 (1994).

${ }^{5}$ D. Vakshoori, R. J. Fischer, M. Hong, D. L. Sivco, G. J. Zydzik, G. N. S. Chu, and A. Y. Cho, Appl. Phys. Lett. 59, 896 (1991).

${ }^{6}$ A. Otomo, Ph.D. dissertation, University of Central Florida, 1995.

${ }^{7}$ A. Otomo, S. Mittler-Neher, G. I. Stegeman, W. H. G. Horsthuis, and G. R. Möhlmann, Electron. Lett. 29, 129 (1993).

${ }^{8}$ R. Normandin and G. I. Stegeman, Opt. Lett. 4, 58 (1979); P. J. Vella, R. Normandin, and G. I. Stegeman, Appl. Phys. Lett. 38, 759 (1981). 